\title{
Low Radioactivity Argon for Dark Matter and Rare Event Searches
}

\section{Dr. Rahaf Ajaj}

Carleton University

1125 Colonel By Drive, Ottawa ON K1S $5 B 6$

E-mail: rahaf.ajaj@carleton.ca

\section{For the Global Argon Dark Matter Collaboration}

The DarkSide-50 dark matter search experiment demonstrated that Argon derived from deep underground sources can be highly reduced in ${ }^{39} \mathrm{Ar}$, and since then the demand for this commodity has risen. Several fundamental physics experiments require Argon reduced in ${ }^{39} \mathrm{Ar}$ as well as ${ }^{42} \mathrm{Ar}$, as well as other rising needs in other scientific fields (e.g., age-dating). With the expanded needs come the questions of availability and how to approach the challenges associated with its production and characterization.

This talk will provide a global picture of low-radioactivity underground Argon procurement, from its production to quality control and quality assurance. We will detail the DarkSide-20k plan for extracting more Argon from the DarkSide-50 source through a project called Urania, as well as another project which will serve to isotopically separate ${ }^{39} \mathrm{Ar}$ from ${ }^{40} \mathrm{Ar}$, called Aria. Finally, DART is a small $(\sim 1 \mathrm{~L})$ chamber that will measure the depletion factor of ${ }^{39} \mathrm{Ar}$ in UAr. The detector will be immersed in the LAr active volume of ArDM (LSC, Spain), which will act as a veto for gammas, allowing a precise measurement of the ${ }^{39} \mathrm{Ar}$ residual activity. 


\section{Introduction}

The DarkSide program is a union of $\sim 420$ researchers from 62 institutions and 14 countries committed to direct searches of dark matter based on the double phase liquid Argon time projection chamber (TPC) technology. On September 2017, the program announced the formation of the Global Argon Dark Matter Collaboration (GADMC). The target was to join the efforts to improve the dark matter discovery potential with background free detectors which will have the capability of reaching the neutrino floor and have the competency to perform very precise measurement of higher-energy solar neutrinos and supernova burst neutrinos.

\section{Underground Argon}

Argon is a promising technology. Besides being a powerful scintillator, it is considered an excellent medium for detection of ionization of WIMP dark matter. In addition, it provides very good pulse shape discrimination between the nuclear recoil signals and electromagnetic background. Based on DEAP3600 data, this rejection factor will surpass $10^{9}$ [1]. Furthermore, it has excellent sensitivity for WIMP masses with single- phase and two-phase detection.

Although Argon has exceptional radio-purity and chemistry, low radon backgrounds at the level of $160 \mathrm{nBq} / \mathrm{kg}$ of ${ }^{222} \mathrm{Rn}, 2.6 \mathrm{nBq} / \mathrm{kg}$ of ${ }^{220} \mathrm{Rn}$ as in DEAP [2], and ${ }^{39} \mathrm{Ar}$ still pose a challenge for current and future experiments. ${ }^{39} \mathrm{Ar}$ is a long half-life radioactive source (269 years) (Q value of $565 \mathrm{KeV}$ ), which emits beta radiation and has an abundance in nature of $1 \mathrm{~Bq} / \mathrm{Kg}$ [3].

The ${ }^{39} \mathrm{Ar}$ is produced by cosmic ray interactions. The concentration of ${ }^{39} \mathrm{Ar}$ underground is lower because gas coming from the mantel of earth has the lowest ${ }^{39} \mathrm{Ar}$ production [4]. DarkSide-50 is the first detector to operate using low radioactivity underground Argon (UAr) extracted in Colorado and purified at Fermilab over a three-year period. The activity of ${ }^{39} \mathrm{Ar}$ for the UAr was found to be $0.73 \mathrm{mBq} / \mathrm{Kg}$, which means that DS-50 measured a depletion factor of 1400 with respect to atmospheric Argon (AAr) [5]. As the future experiments will need to scale the production of UAr greatly to get the mass needed for the, an effort within the GADMC hs been made to build a plant for extraction and purification of UAr for their future experiments.

\section{Future Projects \& Experiments}

The GADMC has established a roadmap for achieving the ultimate sensitivity to the detection of WIMP dark matter using liquid Argon TPCs. The next step will be 20 tonne fiducial volume, dual-phase DS-20k detector located at LNGS, followed by the yet to be defined multi-hundred tonne ARGO detector which will be housed at SNOLAB. To maximize the dark matter discovery potential with background free detectors, the GADMC is working on the increase of the production of UAr (Urania), applying further purification after the extraction of UAr (ARIA), and

targeting further ${ }^{39} \mathrm{Ar}$ reduction for future experiments (ARGO). Subsequently, sensitivity reaching beyond that corresponding to the neutrino floor could be forthcoming at both high and low mass WIMP masses. 


\subsection{Urania}

The collaboration is planning to provide a plant capable of extracting and purifying a total of 330 $\mathrm{kg} /$ day of low radioactivity UAr with a purity of $99.99 \%$. The UAr will be extracted from a $\mathrm{CO}_{2}$ well in Cortez, Colorado, the same location where the collaboration extracted the UAr for DS-50. Urania will procure UAr for the DS-20K experiment planned to start operation at LNGS and for the future ARGO experiment. Urania feed gas will be composed of $95 \% \mathrm{CO}_{2}$, a few percent $\mathrm{N}_{2}$, one percent $\mathrm{CH}_{4}, 430 \mathrm{ppm}$ UAr and some traces of hydrocarbons. The Urania design is not yet released; however, it will be likely including an extraction and purification unit. The extraction unit will reject the bulk of heavy gases from the well $\left(\mathrm{CO}_{2}\right)$. The purification until will remove residual traces of heavy gasses $\left(\mathrm{CO}_{2}, \mathrm{CH}_{4}\right)$, as well as the light gasses $\left(\mathrm{N}_{2}, \mathrm{He}\right)$. Urania will be ready for operation in October 2021 and the first 60 tonnes of UAr production that will be used for DS-20k is expected to be in-hand by the end of July 2022.

As a diagnostic tool during plant construction and for quality assurance for the final gas production, Carleton University is working on assembling, testing and calibrating a multi inlet residual gas analysis (RGA) system for remote operation to be installed and commissioned on site at the Urania plant. Once the final design of the plant is approved, real time monitoring at various output streams at various stages of the gas separation and purification will be defined, and eventually sampled and analyzed by RGA. The system will contain automatically closing valves with a threshold setting in addition to setting for sampling frequency.

To minimize the cosmic activation of the Argon, the transportation of UAr will be moved by land and sea transport modes. The UAr will be transported in high-pressure gas cylinders which allow storage of Argon for years at pressure up to 400 bar. Each shipment will be able to carry approximately 3-5 tonnes of UAr. The transportation project will contain two phases. Phase one to ship 60 tonnes of UAr from URANIA to ARIA for further chemical purification and then to the final destination of LNGS in Italy. The second phase is to ship 400 tonnes of UAr to be stored at SNOLAB in Canada for future use in the ARGO detector.

\subsection{ARIA}

For further purification of the UAr, the GADMC will be using ARIA which will first be a 350meter-tall cryogenic distillation column called Seruci-I. It is a facility to provide detector grade Ar, free from $\mathrm{N}$ and other impurities and will provide the final chemical separation for the DS$20 \mathrm{k}$ target. ARIA is ultimately being built for the isotopic separation of ${ }^{39} \mathrm{Ar}$ from ${ }^{40} \mathrm{Ar}$ at a rate of several $\mathrm{kg}$ per day and at a purity of $99 \%$. ARIA is currently in a testing and construction phase, currently using only a small portion of the final column which will contain over one thousand equilibrium separation stages. The commissioning of the Seruci-I column is scheduled by the second quarter of 2020 in Sardinia, Italy. 


\subsection{ARGUS}

To avoid the long-term exposure of the UAr on the surface and potential activation of ${ }^{39} \mathrm{Ar}$, the GADMC are planning for a long-term underground storage facility. The storage facility (called ARGUS) is planned to be at SNOLAB. ARGUS is expected to store a total of 300 high-pressure gas cylinders which will contain the more than 400 tonnes of UAr required for ARGO.

\subsection{DarkSide-20K}

The next generation of LAr dark matter detectors will consist of two detectors (inner \& veto). The inner detector is a dual-phase Argon time projection chamber (LAr TPC) and is fully concealed within an ultra-pure acrylic (PMMA) vessel. DS-20K will be operating with about 60 tonnes of low activity UAr in total, but can be fiducialized to provide extremely good sensitivity with just the central 20 tonnes. It is designed to to achieve an exposure of 100 tonne-year in a backgroundfree mode (less than 0.1 events of non-neutrino background). DS-20K will be located at LNGS and commissioned by 2023 .

\subsection{DArT}

The depletion factor of ${ }^{39} \mathrm{Ar}$ in the UAr relative to AAr, coming from both URANIA and ARIA, will be measured by a small TPC chamber called DArT. It will be immersed in the active LAr volume of ArDM at LSC by the end of 2019, with ArDM acting as a veto to amplify the sensitivity of the smaller DArT device.

\subsection{ARGO}

The GADMC is aiming to build the most sensitive WIMP search by reaching beyond the sensitivity corresponding to the neutrino floor in dark matter searches. ARGO will be operating with about 400 tonnes of UAr with a detector free of background. The GADMC is planning for a 300 tonne fiducial volume in order to achieve an exposure of 3 ktonne-year free of background in the WIMP search region. The detailed design is currently under study and assessment including experience from current both S1 - only and dual phase TPC experiments is being taken into account. The conceptual design is underway to take into account backgrounds such as cosmogenic-related ones, neutrons and Cherenkov events, etc. ARGO is expected to allow very sensitive measurements for intermediate-energy solar neutrino and will also provide excellent measurement of the neutral current channel for neutrinos coming from a galactic supernova burst. Further possibility of other physics measurements is under assessment by reconfiguring the detector, such as a potential measurement for neutrino less double beta decay. It is projected that the detector will be commissioned by 2029 . 


\section{References}

[1] Amaudruz, P. A., Baldwin, M., Batygov, M., Beltran, B., Bina, C. E., Bishop, D., ... \& Bromwich, T. (2018). First results from the DEAP-3600 dark matter search with argon at SNOLAB. Physical review letters, 121(7), 071801.

[2] Amaudruz, P. A., Batygov, M., Beltran, B., Boudjemline, K., Boulay, M. G., Cai, B., ... \& Contreras, D. (2015). Radon backgrounds in the DEAP-1 liquid-argon-based Dark Matter detector. Astroparticle Physics, 62, 178-194.

[3] Loosli, H. H., \& Oeschger, H. (1968). Detection of 39Ar in atmospheric argon. Earth and Planetary Science Letters, 5, 191-198.

[4] Šrámek, O., Stevens, L., McDonough, W. F., Mukhopadhyay, S., \& Peterson, R. J. (2017). Subterranean production of neutrons, $39 \mathrm{Ar}$ and $21 \mathrm{Ne}$ : Rates and uncertainties. Geochimica et Cosmochimica Acta, 196, 370-387.

[5] P. Anges et al. DarkSide Collaboration. Results from the first use of low radioactivity argon in a dark matter search. Phys. Rev. D 95, 069901 (2017). 